\title{
NECESSARY CONDITIONS FOR EQUIVALENCE WITH THE SPECIAL THEORY OF RELATIVITY
}

\author{
A. Sfarti \\ University of California, Berkeley (Soda Hall), Berkeley, California, USA \\ E-mail: egas@pacbell.net
}

Received 4 August 2009; revised 18 December 2009; accepted 18 December 2009

\begin{abstract}
The Mansouri-Sexl theory is a well known test theory of relativity. Tangherlini [1] produced a theory that is a limit case for the Mansouri-Sexl theory. We will show that Tangherlin's theory disagrees with the waveguide theory, predicting a different result than special relativity (SR), so, the Tangherlini theory is not equivalent with SR. We will also show that for a theory to be equivalent to the special relativity, contrary to Robertson [2] it is not sufficient to predict the same results for the Michelson-Morley, Kennedy-Thorndike, and Ives-Stilwell experiments, it must also predict the same results for a waveguidebased experiment described in Section 4 of our paper.
\end{abstract}

Keywords: Mansouri-Sexl test theory, SME, Tangherlini theory, Ives-Stilwell experiment, transverse Doppler effect, waveguide theory

PACS: $03.30 .+\mathrm{p}$

\section{The Mansouri-Sexl test theory}

Various test theories differ in their assumptions about the form of the Lorentz transforms. The main test theories of special relativity (SR) are named after their authors, Mansouri and Sexl [3-5]. These test theories can also be used to examine potential alternative theories to SR - such alternative theories predict particular values of the parameters of the test theory, which can easily be compared to values determined by experiments analysed with the test theory.

\section{The Mansouri-Sexl theory of the Doppler effect}

In his 1905 paper, "On the Electrodynamics of Moving Bodies" [6], Einstein produces an interesting blueprint for deriving the general formula for the Doppler effect. He starts by considering a generic electromagnetic wave of phase $\Phi$, frequency $\nu-\omega /(2 \pi)$, and of wave-vector $(l, m, n)$ propagating with speed $c$ towards the origin $O$ of a frame $K$. From the perspective of a system $K$, of coordinates $(x, y, z, t)$ the phase is

$$
\Phi=\omega\left(t-\frac{l x+m y+n z}{c}\right)
$$

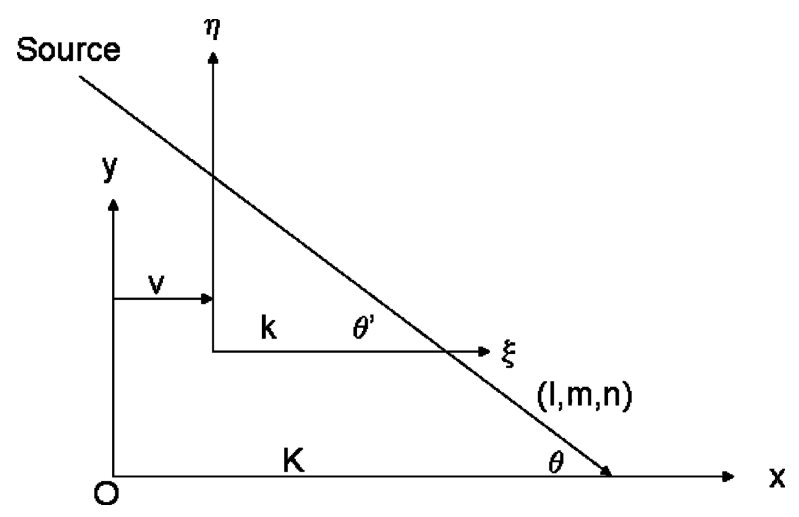

Fig. 1. Reference frames $K$ and $k$.

Let $k$ be a system moving with the speed $v$ along the positive $x$ axis of $K$ (see Fig. 1). We want to determine the form of the phase from the perspective of $k$, departing from the light source. Since $K$ and $k$ are in a translation motion along the $x$ axis with respect to each other we replace the Lorentz transformations in Einstein derivation:

$$
\begin{aligned}
& \xi=\gamma(x-v t), \quad \psi=y, \quad \zeta=z, \\
& \tau=\gamma\left(t-\frac{v x}{c^{2}}\right), \quad \gamma=\frac{1}{\sqrt{1-v^{2} / c^{2}}},
\end{aligned}
$$


with the corresponding Mansouri-Sexl transforms

$$
\begin{aligned}
& x=b(v)(X-v T), \quad y=d(v) Y, \quad z=d(v) Z \\
& t=(a-b \varepsilon v) T+b \varepsilon X .
\end{aligned}
$$

The Tangherlini transforms [1,7-9] are a particular case of the Mansouri-Sexl transforms in the case

$$
b=\gamma, \quad a=1 / \gamma, \quad \varepsilon=0, \quad d=1 .
$$

In this particular case the Mansouri-Sexl transforms (3) become the Tangherlini transforms:

$$
\begin{aligned}
& x=\gamma(v)(X-v T), \quad y=Y, \quad z=Z, \\
& t=T / \gamma(v) .
\end{aligned}
$$

We stress once again that the Tangherlini theory is not a test theory of SR, it is just one of the many theories claimed to be equivalent to SR. In (3) $(X, Y, Z, T)$ represent the coordinates in the preferential frame $\Sigma$ while $(x, y, z, t)$ are the corresponding coordinates in the lab frame $S . c^{\prime}$ is the (anisotropic) light speed in $S$ while $c$ is the isotropic light speed in $\Sigma$. In the preferential frame $\Sigma$

$$
\Phi=\omega\left(T-\frac{l X+m Y+n Z}{c}\right) .
$$

In the lab frame $S$, the phase $\phi$ must have a form similar to the form (6):

$$
\begin{aligned}
\phi= & \omega^{\prime}\left(t-l^{\prime} \frac{x}{c^{\prime}}-m^{\prime} \frac{y}{c^{\prime}}-n^{\prime} \frac{z}{c^{\prime}}\right)=\omega^{\prime}[(a-b \varepsilon v) T+ \\
& \left.+b \varepsilon X-\frac{b l^{\prime}}{c^{\prime}}(X-v T)-\frac{d m^{\prime}}{c^{\prime}} Y-\frac{d n^{\prime}}{c^{\prime}} Z\right]= \\
= & \omega^{\prime}\left(a-b \varepsilon v+\frac{b v l^{\prime}}{c^{\prime}}\right)\left[T-\left(a-b \varepsilon v+\frac{b v l^{\prime}}{c^{\prime}}\right)^{-1} \times\right. \\
& \times c b\left(\frac{l^{\prime}}{c^{\prime}}-\varepsilon\right) \frac{X}{c}-\left(a-b \varepsilon v+\frac{b v l^{\prime}}{c^{\prime}}\right)^{-1} \frac{d m^{\prime} Y}{c}- \\
& \left.-\left(a-b \varepsilon v+\frac{b v l^{\prime}}{c^{\prime}}\right)^{-1} \frac{d n^{\prime} Z}{c}\right] .
\end{aligned}
$$

In the lab frame, by comparing (6) with (7) we obtain the Mansouri-Sexl aberration formula and the Doppler shifted frequency for a receding light source $[10,11]$ :

$$
\omega_{\mathrm{R}}^{\prime}=\omega \frac{1-\frac{v}{c} \cos \theta}{a},
$$

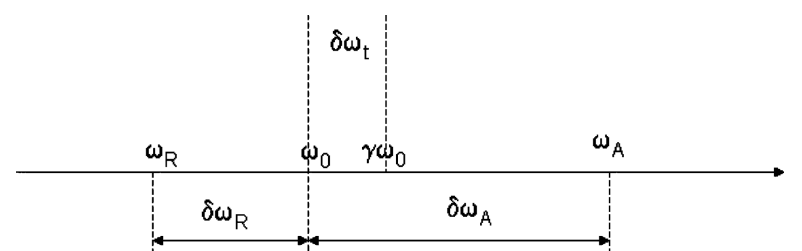

Fig. 2. The Ives-Stilwell measurements.

and, for approaching the light source

$$
\omega_{\mathrm{A}}^{\prime}=\omega \frac{1+\frac{v}{c} \cos \theta}{a} .
$$

The formula

$$
\omega^{\prime}=\omega \Gamma(1 \pm \beta \cos \theta)
$$

represents the general formula for the Mansouri-Sexl Doppler effect measured in the lab frame $S$ where $\Gamma(v)=1 / a(v)$ and $\beta=v / c$, and $c$ is the isotropic light speed in $\Sigma$. A quick sanity check shows that in SR $a=1 / \gamma$ resulting into $\Gamma=\gamma$ and so $\omega^{\prime}=$ $\omega \gamma[1-(v / c) \cos \theta]$, in perfect agreement with Einstein's derivation [6]. For the case of the Tangherlini theory $\Gamma(v)=\gamma(v)$ and the Tangherlini Doppler effect is identical with the special relativity one. Therefore, the Tangherlini theory will predict the same outcome as special relativity (SR) for the Ives-Stilwell experiment.

\section{The Ives-Stilwell experiment}

As seen in [6], the general form of the longitudinal Doppler effect, where the emitter path makes an angle $\theta$ with the line of sight is

$$
\omega_{\mathrm{R}}=\gamma \omega_{0}(1-\beta \cos \theta)<\gamma \omega_{0}
$$

for the receding wave (red shift) and

$$
\begin{aligned}
\omega_{\mathrm{A}}= & \gamma \omega_{0}[1-\beta \cos (\theta+\pi)]= \\
& =\gamma \omega_{0}(1+\beta \cos \theta)>\gamma \omega_{0}
\end{aligned}
$$

for the approaching wave (blue shift).

Substituting $\theta=\pi / 2$ in (11) we obtain the transverse Doppler effect (TDE) formula $\omega_{\mathrm{t}}=\gamma \omega_{0}$. The problem is that for low ion speeds $\omega_{t}$ is very close to $\omega_{0}$. Ives and Stilwell came up with a very clever scheme of separating the very small transverse effect directly from the much larger longitudinal effect. Let

$$
\delta \omega_{\mathrm{t}}=\gamma \omega_{0}-\omega_{0}
$$


Looking at Fig. 2 let $\delta \omega_{\mathrm{R}}$ be the total red shift of the receding wave and let $\delta \omega_{\mathrm{A}}$ be the total blue shift of the approaching wave with respect to the reference $\omega_{0}$. Then

$$
\begin{aligned}
& \delta \omega_{\mathrm{A}}=\left(\omega_{\mathrm{A}}-\gamma \omega_{0}\right)+\delta \omega_{\mathrm{t}}=\beta \gamma \omega_{0} \cos \theta+\delta \omega_{\mathrm{t}}, \\
& \delta \omega_{\mathrm{R}}=\left(\gamma \omega_{0}-\omega_{\mathrm{R}}\right)-\delta \omega_{\mathrm{t}}=\beta \gamma \omega_{0} \cos \theta-\delta \omega_{\mathrm{t}} .
\end{aligned}
$$

Ives and Stilwell could measure on a spectrograph the quantities $\delta \omega_{\mathrm{R}}$ and $\delta \omega_{\mathrm{A}}$ because they represent the deviation from the reference value $\omega_{0}$. From (14) and (15) they obtained

$$
\delta \omega_{\mathrm{t}}=\frac{\delta \omega_{\mathrm{A}}-\delta \omega_{\mathrm{R}}}{2} .
$$

By taking the sum

$$
\delta \omega_{\mathrm{A}}+\delta \omega_{\mathrm{R}}=2 \beta \gamma \omega_{0} \cos \theta,
$$

Ives and Stilwell calculated the speed $v$ of the ions from

$$
\beta \gamma=\frac{\delta \omega_{\mathrm{A}}+\delta \omega_{\mathrm{R}}}{2 \omega_{0} \cos \theta}
$$

Arranging for $\theta$ to be very close to zero they obtained

$$
\frac{u / c}{\sqrt{1-(u / c)^{2}}}=\frac{\delta \omega_{\mathrm{A}}+\delta \omega_{\mathrm{R}}}{2 \omega_{0}} .
$$

Solving (19) for $u$ Ives and Stilwell produced the graphs $\delta \omega_{\mathrm{t}}$ as a function of the ions' speed $u$, a very clever way of separating the transverse Doppler effect (TDE) from an experiment that is essentially longitudinal.

\section{The Tangherlini theory for electromagnetic wave propagation in waveguides}

An earlier paper by Gagnon [12] makes clever use of the Earth's revolution around the Sun and of the Earth's diurnal rotation. Let $\boldsymbol{v}$ represent the Earth revolution speed. Let $\Sigma(\xi, \psi, \zeta)$ represent the preferential reference frame from the Tangherlini theory. In the lab frame, according to [13] the Tangherlini transformation of the standard [14] wave equation $\nabla^{2} E=$ $\left(1 / c^{2}\right)\left(\partial^{2} E / \partial t^{2}\right)$ is

$$
\nabla^{2} E-\frac{2}{c^{2}}(\boldsymbol{v} \cdot \nabla) \frac{\partial E}{\partial t}-\left(1-\frac{v^{2}}{c^{2}}\right) \frac{1}{c^{2}} \frac{\partial^{2} E}{\partial t^{2}}=0,
$$

where $E=E(x, y, z, t)=u_{x} E_{x}+u_{y} E_{y}+u_{z} E_{z}$. From wave theory we know that the solution for equation (20) is of the form

$$
E_{z}=X(x) Y(y) \mathrm{e}^{\mathrm{i}(k z-\omega t)},
$$

with the boundary condition

$E_{z}(x=0)=E_{z}(x=a)=E_{z}(y=0)=E_{z}(y=b)=0$.

Let $X(x)$ and $Y(y)$ be two functions continuous with continuous second order derivatives. The problem is now reduced to finding the solution for the differential equation

$$
\begin{aligned}
0= & Y \frac{\mathrm{d}^{2} X}{\mathrm{~d} x^{2}}+X \frac{\mathrm{d}^{2} Y}{\mathrm{~d} y^{2}}+X Y\left[-k^{2}+2 \frac{v_{z} \omega}{c^{2}} k+(1-\right. \\
& \left.\left.-\frac{v^{2}}{c^{2}}\right) \frac{\omega^{2}}{c^{2}}\right]-\mathrm{i} \frac{2 \omega}{c^{2}}\left(v_{x} Y \frac{\mathrm{d} X}{\mathrm{~d} x}+v_{y} X \frac{\mathrm{d} Y}{\mathrm{~d} y}\right),
\end{aligned}
$$

with the boundary conditions

$$
X(0)=X(a)=0, \quad Y(0)=Y(b)=0,
$$

where $v^{2}=v_{x}^{2}+v_{y}^{2}+v_{z}^{2}$.

Let

$$
C=-k^{2}+2 \frac{v_{z} \omega}{c^{2}} k+\left(1-\frac{v^{2}}{c^{2}}\right) \frac{\omega^{2}}{c^{2}} .
$$

Assuming $X Y \neq 0$ we can divide expression (23) by $X Y$ :

$$
\begin{aligned}
-C= & \left(\frac{1}{X} \frac{\mathrm{d}^{2} X}{\mathrm{~d} x^{2}}-\frac{2 \mathrm{i} \omega v_{x}}{c^{2}} \frac{1}{X} \frac{\mathrm{d} X}{\mathrm{~d} x}\right)+ \\
& +\left(\frac{1}{Y} \frac{\mathrm{d}^{2} Y}{\mathrm{~d} y^{2}}-\frac{2 \mathrm{i} \omega v_{y}}{c^{2}} \frac{1}{Y} \frac{\mathrm{d} Y}{\mathrm{~d} y}\right) .
\end{aligned}
$$

Since $X(x)$ is a function only of $x$ and $Y(y)$ is a function only of $y$ and since the left-hand side of (26) is a constant it results immediately that

$$
\begin{gathered}
\frac{1}{X} \frac{\mathrm{d}^{2} X}{\mathrm{~d} x^{2}}-\frac{2 \mathrm{i} \omega v_{x}}{c^{2}} \frac{1}{X} \frac{\mathrm{d} X}{\mathrm{~d} x}=-\alpha, \\
\frac{1}{Y} \frac{\mathrm{d}^{2} Y}{\mathrm{~d} y^{2}}-\frac{2 \mathrm{i} \omega v_{y}}{c^{2}} \frac{1}{Y} \frac{\mathrm{d} Y}{\mathrm{~d} y}=-\beta .
\end{gathered}
$$

Equation

$$
\frac{\mathrm{d}^{2} X}{\mathrm{~d} x^{2}}-\frac{2 \mathrm{i} \omega v_{x}}{c^{2}} \frac{\mathrm{d} X}{\mathrm{~d} x}+\alpha X=0
$$

must have a solution of the type

$$
X(x)=\mathrm{e}^{\mathrm{i} r x},
$$


i. e. two differential equations of degree two with imaginary coefficients follow, each producing the characteristic equation

$$
\begin{aligned}
& -r^{2}+2 \frac{v_{x}}{c} \frac{\omega}{c} r+\alpha=0, \text { where } \\
& r_{1,2}=\frac{v_{x}}{c} \frac{\omega}{c} \pm \sqrt{\left(\frac{v_{x}}{c} \frac{\omega}{c}\right)^{2}+\alpha}, \\
& X(x)=C_{1} \mathrm{e}^{\mathrm{i} r_{1} x}+C_{2} \mathrm{e}^{\mathrm{i} r_{2} x}=\mathrm{e}^{\mathrm{i} x \frac{v_{x}}{c} \frac{\omega}{c}} \times \\
& \times\left(C_{1} \mathrm{e}^{\mathrm{i} x \sqrt{\alpha+\left(\frac{v_{x}}{c} \frac{\omega}{c}\right)^{2}}}+C_{2} \mathrm{e}^{-\mathrm{i} x \sqrt{\alpha+\left(\frac{v_{x}}{c} \frac{\omega}{c}\right)^{2}}}\right) . \\
& 0=X(0)=C_{1}+C_{2} \text { implies } \\
& C_{2}=-C_{1},
\end{aligned}
$$

therefore

$$
\begin{aligned}
X(x)= & \mathrm{e}^{\mathrm{i} x \frac{v_{x}}{c} \frac{\omega}{c}} C_{1} \times \\
& \times\left(\mathrm{e}^{\mathrm{i} x \sqrt{\alpha+\left(\frac{v_{x}}{c} \frac{\omega}{c}\right)^{2}}}-\mathrm{e}^{-\mathrm{i} x \sqrt{\alpha+\left(\frac{v_{x}}{c} \frac{\omega}{c}\right)^{2}}}\right), \\
0= & X(a)=\mathrm{e}^{\mathrm{i} a \frac{v_{x}}{c} \frac{\omega}{c}} C_{1} \times \\
& \times\left(\mathrm{e}^{\mathrm{i} a \sqrt{\alpha+\left(\frac{v_{x}}{c} \frac{\omega}{c}\right)^{2}}}-\mathrm{e}^{-\mathrm{i} a \sqrt{\alpha+\left(\frac{v_{x}}{c} \frac{\omega}{c}\right)^{2}}}\right), \\
0= & 2 \mathrm{i} \sin \left[a \sqrt{\alpha+\left(\frac{v_{x}}{c} \frac{\omega}{c}\right)^{2}}\right], \\
& a \sqrt{\alpha+\left(\frac{v_{x}}{c} \frac{\omega}{c}\right)^{2}}=m \pi, \\
& \alpha=\left(\frac{m \pi}{a}\right)^{2}-\left(\frac{v_{x}}{c} \frac{\omega}{c}\right)^{2} .
\end{aligned}
$$

Similarly,

$$
\begin{aligned}
Y(y)= & \mathrm{e}^{\mathrm{i} y \frac{v_{y}}{c} \frac{\omega}{c}} C_{3} \times \\
& \times\left(\mathrm{e}^{\mathrm{i} y \sqrt{\beta+\left(\frac{v_{y}}{c} \frac{\omega}{c}\right)^{2}}}-\mathrm{e}^{-\mathrm{i} y \sqrt{\beta+\left(\frac{v_{y}}{c} \frac{\omega}{c}\right)^{2}}}\right), \\
\beta & =\left(\frac{n \pi}{b}\right)^{2}-\left(\frac{v_{y}}{c} \frac{\omega}{c}\right)^{2} .
\end{aligned}
$$

Thus, from previous definitions it follows that

$$
\begin{aligned}
& -k^{2}+2 \frac{v_{z} \omega}{c^{2}} k+\left(1-\frac{v^{2}}{c^{2}}\right) \frac{\omega^{2}}{c^{2}}=C=\alpha+\beta, \\
& k^{2}-2 \frac{v_{z} \omega}{c^{2}} k-\left(1-\frac{v^{2}}{c^{2}}\right) \frac{\omega^{2}}{c^{2}}+(\alpha+\beta)=0, \\
& k^{2}-2 \frac{v_{z} \omega}{c^{2}} k-\left(1-\frac{v^{2}}{c^{2}}\right) \frac{\omega^{2}}{c^{2}}+\left(\frac{m \pi}{a}\right)^{2}+\left(\frac{n \pi}{b}\right)^{2}- \\
& -\left(\frac{v_{x}}{c} \frac{\omega}{c}\right)^{2}-\left(\frac{v_{y}}{c} \frac{\omega}{c}\right)^{2}=0, \\
& k^{2}-2 \frac{v_{z} \omega}{c^{2}} k-\left(1-\frac{v_{z}^{2}}{c^{2}}\right) \frac{\omega^{2}}{c^{2}}+\frac{\omega_{m n}^{2}}{c^{2}}=0,
\end{aligned}
$$

where

$$
\frac{\omega_{m n}^{2}}{c^{2}}=\left(\frac{m \pi}{a}\right)^{2}+\left(\frac{n \pi}{b}\right)^{2} .
$$

Solving (44) for $k$ we obtain

$$
k\left(\omega, v_{z}\right)=\frac{v_{z}}{c} \frac{\omega}{c} \pm \frac{1}{c} \sqrt{\omega^{2}-\omega_{m n}^{2}} .
$$

$k$ is a real number if and only if $\omega \geq \omega_{m n}, \omega_{m n}$ is the "cutoff pulsation" below which $k$ becomes imaginary and the wave attenuates instead of propagating properly to the end of the waveguide.

$$
\begin{aligned}
E_{z}= & B \sin \left(\frac{m \pi}{a} x\right) \sin \left(\frac{n \pi}{b} y\right) \times \\
& \times \cos \left[k z+\frac{v_{x}}{c} \frac{\omega}{c} x+\frac{v_{y}}{c} \frac{\omega}{c} y-\omega t\right] .
\end{aligned}
$$

There is a second waveguide in the experiment, driven at the same pulsation $\omega$ but with a very different "cutoff" pulsation $\omega_{p q}$, we can write immediately the electrical field:

$$
\begin{aligned}
E_{z}^{\prime}= & B^{\prime} \sin \left(\frac{p \pi}{a^{\prime}} x\right) \sin \left(\frac{q \pi}{b^{\prime}} y\right) \times \\
& \times \cos \left[k z^{\prime}+\frac{v_{x}}{c} \frac{\omega}{c} x+\frac{v_{y}}{c} \frac{\omega}{c} y-\omega t\right],
\end{aligned}
$$

with

$$
\frac{\omega_{p q}^{2}}{c^{2}}=\left(\frac{p \pi}{a^{\prime}}\right)^{2}+\left(\frac{q \pi}{b^{\prime}}\right)^{2}
$$




$$
k^{\prime}\left(\omega, v_{z}\right)=\frac{v_{z}}{c} \frac{\omega}{c} \pm \frac{1}{c} \sqrt{\omega^{2}-\omega_{p q}^{2}} .
$$

We have enough degrees of freedom in selecting the geometries of the waveguides such that

$$
\begin{aligned}
& B^{\prime} \sin \left(\frac{p \pi}{a^{\prime}} x\right) \sin \left(\frac{q \pi}{b^{\prime}} y\right)= \\
& =B \sin \left(\frac{m \pi}{a} x\right) \sin \left(\frac{n \pi}{b} y\right)=E_{0}, \\
& E_{z}=E_{0} \times \\
& \times \cos \left[a_{m n} z+\frac{v_{x}}{c} \frac{\omega}{c} x+\frac{v_{y}}{c} \frac{\omega}{c} y+\frac{v_{z}}{c} \frac{\omega}{c} z-\omega t\right], \\
& E_{z}^{\prime}=E_{0} \times \\
& \times \cos \left[a_{p q} z+\frac{v_{x}}{c} \frac{\omega}{c} x+\frac{v_{y}}{c} \frac{\omega}{c} y+\frac{v_{z}}{c} \frac{\omega}{c} z-\omega t\right], \\
& a_{m n}=-\frac{1}{c} \sqrt{\omega^{2}-\omega_{m n}^{2}}, \\
& a_{p q}=-\frac{1}{c} \sqrt{\omega^{2}-\omega_{p q}^{2}} .
\end{aligned}
$$

The phase difference between $E_{z}$ and $E_{z}^{\prime}$ is

$$
\Delta \Phi=\left(a_{m n}-a_{p q}\right) z .
$$

The two waveguides in the experiment have different cutoff pulsations [12] so the expression (56) is nonnull. By contrast, special relativity predicts $\Delta \Phi=0$ for the Gagnon experiment [12], that is, light speed is isotropic. The Tangherlini theory predicts a non-null one way light speed anisotropy contrary to the null prediction in SR. Thus, the Tangherlini theory is not equivalent to SR.

\section{Conclusion}

We have demonstrated that Tangherlini's theory, while producing identical predictions with SR for the Ives-Stilwell experiment produces different predictions in the case of electromagnetic wave propagation in waveguides. Thus, the Tangherlini theory is not equivalent to SR. Contrary to Robertson [2], the fact that the Tangherlini theory passes the experiments of MichelsonMorley, Kennedy-Thorndike and Ives-Stilwell is not sufficient to make the Tangherlini theory indistinguish-

able from SR since it predicts a different result for the electromagnetic-type experiments suggested in [10$13,15,16]$.

\section{References}

[1] F.R. Tangherlini, An introduction to the general theory of relativity, Nuovo Cimento Supp. 20, 1 (1961).

[2] H.P. Robertson, Postulate versus observation in the special theory of relativity, Rev. Mod. Phys. 21, (1949).

[3] R. Mansouri and S.U. Sexl, A test of special relativity, Gen. Rel. Grav. 8, 497 (1977).

[4] R. Mansouri and S.U. Sexl, A test of special relativity, Gen. Rel. Grav. 8, 515 (1977).

[5] R. Mansouri and S.U. Sexl, A test of special relativity, Gen. Rel. Grav. 8, 809 (1977).

[6] A. Einstein, On the electrodynamics of moving bodies, Ann. Phys. 17 (1905).

[7] G.B. Malykin, Para-Lorentz transformations, Phys. Usp. 53, 263 (2009).

[8] A. Eagle, A criticism of special relativity, Philos. Mag. Ser. 26, 410 (1938).

[9] A. Eagle, Note on synchronizing clocks in moving systems, Philos. Mag. Ser. 28, 592 (1939).

[10] A. Sfarti, One way light speed measurement - experimental proof of light speed isotropy, in: Proceedings of the XI Marcel Grossman Conference, Berlin (2006).

[11] A. Sfarti, Detection of light speed anisotropy via a highspeed Ives-Stillwell experiment, Can. J. Phys. 86, 5, 747 (2008).

[12] D.R. Gagnon, D.G. Torr, P.T. Kolen, and T. Chang, Guided-wave measurement of the one-way speed of light, Phys. Rev. A 38, 4 (1988).

[13] T. Chang, Maxwell's equations in anisotropic space, Phys. Lett. 70A, 1 (1979).

[14] S. Wentworth, Fundamentals of Electromagnetics with Engineering Applications, (Wiley, 2006) p. 338-355.

[15] T. Chang and D. Torr, Dual properties of spacetime under an alternative Lorentz transformation, Found. Phys. Lett. 1, 4 (1988).

[16] T. Chang, D. Torr, and D. Gagnon, A modified Lorentz theory as a test theory of special relativity, Found. Phys. Lett. 1, 4 (1988). 


\title{
BŪTINOS EKVIVALENTUMO SPECIALIAJAI RELIATYVUMO TEORIJAI SĄLYGOS
}

\author{
A. Sfarti
}

Kalifornijos universitetas Berklyje, JAV

\section{Santrauka}

Mansouri-Sexl teorija gerai žinoma kaip testinè reliatyvumo teorija. Tangherlini [1] pasiūle teoriją, kuri yra ribinis MansouriSexl teorijos atvejis. Parodyta, kad Tangherlini teorija nesutampa su bangolaidžio teorija, numatydama kitoki rezultatą, nei specialioji reliatyvumo teorija. Taigi, Tangherlini teorija nèra ekvivalenti specialiajai reliatyvumo teorijai. Taip pat parodoma, kad, priešingai nei teigia Robertson [2], būtinos tam tikros teorijos ekvivalentumo specialiajai reliatyvumo teorijai sąlygos nèra tik tụ pačiu rezultatų numatymas Michelson-Morley, Kennedy-Thorndike ir Ives-Stilwell eksperimentuose, bet ir vienodo rezultato prognozé bangolaidžio eksperimente, aprašytame šio straipsnio 4 skyriuje. 